\title{
A Perturbation Finite Element Method for Modeling Electrostatic MEMS without Remeshing
}

\author{
Mohamed Boutaayamou $^{1}$, Ruth V. Sabariego ${ }^{1}$ and Patrick Dular ${ }^{1,2}$ \\ Department of Electrical Engineering and Computer Science, ${ }^{1}$ University of Liège, ${ }^{2}$ FNRS, Belgium \\ mboutaayamou@ulg.ac.be
}

\begin{abstract}
This paper deals with the coupled electrostatic-mechanical analysis of electrostatically actuated MEMS. An iterative perturbation procedure in conjunction with the finite element method is used to solve the coupled problem without the need of remeshing the whole electric domain. The method offers the advantage of overcoming degenerated finite elements in the mesh of some electric regions where the deflection of the MEMS moving parts is critical. The actuation of such systems is achieved by applying either an electric voltage or a global charge.
\end{abstract}

\section{Introduction}

A common configuration of an electrostatic MEMS consists of a flexible electrode suspended over a stationary conductor. One electrode is electrically connected to ground, while the other is driven by a voltage source. When a voltage is applied, electric forces act on the free electrode resulting in structural deformations, which modify the electric field and force distribution. In some cases, the microstructure can undergo large displacements which may cause pull-in instability [1].

The electrostatic-structural problem has already been treated in several works. In [2][3], for example, an automated mesh morphing algorithm has been employed to update the field mesh after structural deformation. Remeshing of all or part of the geometry during displacement implies the need of a reliable automatic mesh generator which provides optimal and homogeneous meshing [4]. If the geometry is relatively complex, such a mesh generator may be time-consuming. In [5][6], the finite element (FE) technique is used to solve the structural problem while the boundary element (BE) method is used to handle the electric problem and allows to obtain the deformation without any remeshing (the air is not meshed). However, the BE electrostatic model leads to a fully populated system matrix which is computationally expensive and may limit the size of the coupled problems to be solved.

In this paper, an iterative perturbation FE method (PFEM) [7]-[9] is used to avoid remeshing in the coupled electrostatic-mechanical analysis of electrostatic MEMS. It is an extension of [9] where only fixed conductors are considered. The perturbation technique uses independent meshes: one for the fixed part of the microstructure and another for the moving part. The solution is transferred from one mesh to the other by means of a projection method [10]. The mesh of the moving part is fine enough around the corners in order to capture strong electric fields and to accurately compute driving forces via the virtual work principal. A structural analysis is then performed to determine the moving part microstructure deflection. The originality of this approach lays on the fact that the moving mesh is not bounded by the fixed one. Degenerated elements of the electric domain mesh in pull-in are thus avoided. 
A cantilever microbeam and a combdrive are considered as test cases. The numerical results of the microbeam are compared with the experimental measurements.

\section{Electrostatic Model of MEMS by the PFEM}

\section{A. Unperturbed and Perturbed Electrostatic Problems-Strong Formulations}

An electrostatic problem is to be solved in a domain $\Omega$, with boundary $\Gamma=\Gamma_{e} \cup \Gamma_{d}$, of the 2-D or 3-D Euclidean space (Fig. 1). The conducting parts of $\Omega$ are denoted $\Omega_{c}$, with boundary $\Gamma_{c}$. The governing differential equations and constitutive law in $\Omega$ are

$$
\operatorname{curl} \boldsymbol{e}=0, \quad \operatorname{div} \boldsymbol{d}=q, \quad \boldsymbol{d}=\varepsilon \boldsymbol{e},
$$

with boundary conditions (BCs)

$$
\boldsymbol{n} \times\left.\boldsymbol{e}\right|_{\Gamma_{e}}=0,\left.\quad \boldsymbol{n} \cdot \boldsymbol{d}\right|_{\Gamma_{d}}=0,
$$

where $\boldsymbol{e}$ is the electric field, $\boldsymbol{d}$ is the electric flux density, $q$ is the electric charge density, $\varepsilon$ is the electric permittivity and $\boldsymbol{n}$ is the unit normal exterior to $\Omega$. From (1a), the electric field $\boldsymbol{e}$ can be derived from an electric scalar potential $v$, i.e., $\boldsymbol{e}=-\operatorname{grad} v$.

Hereafter, the subscripts $u$ and $p$ refer to the unperturbed and perturbed quantities and associated domains, respectively.

In this paper, the electrostatic problem is solved in two steps (Fig. 2). An unperturbed electrostatic problem is first solved in $\Omega$ without considering a moving conductive region $\Omega_{c, p}$. Its solution gives the source for the moving region, i.e., the so-called perturbing region. For each position of the latter, a perturbation problem is solved in a sub-domain $\Omega_{p}$ (i.e., $\Omega_{c, p}$ and its neighborhood). At the discrete level, the mesh of $\Omega_{p}$ is distinct from that of $\Omega$ what allows to benefit from mesh refinement and deformation of different regions.

\section{B. Perturbation Problem}

For each position of the moving perturbing region $\Omega_{c, p}$, a perturbation problem is defined in $\Omega_{p}$. Its related quantities are determined by subtracting the unperturbed equations from the perturbed ones [7]-[9]. The obtained equations are expressed in terms of the field distortions $\boldsymbol{e}=\boldsymbol{e}_{p}-\boldsymbol{e}_{u}$ and $\boldsymbol{d}=\boldsymbol{d}_{p}-\boldsymbol{d}_{u}$ as

$$
\begin{gathered}
\operatorname{curl} \boldsymbol{e}=0, \operatorname{div} \boldsymbol{d}=0, \boldsymbol{d}=\varepsilon_{p} \boldsymbol{e}+\left(\varepsilon_{p}-\varepsilon_{u}\right) \boldsymbol{e}_{u}, \\
\boldsymbol{n} \times\left.\boldsymbol{e}\right|_{\Gamma_{e, p}}=\boldsymbol{n} \times \boldsymbol{e}_{s},\left.\quad \boldsymbol{n} \cdot \boldsymbol{d}\right|_{\Gamma_{d, p}}=\boldsymbol{n} \cdot \boldsymbol{d}_{s} .
\end{gathered}
$$

In (3b), it is assumed that there is no volume charge density in $\Omega_{p}$. BCs (4a-b) on the outer boundary of $\Omega_{p}$ are first defined as homogeneous, i.e., $\boldsymbol{n} \times \boldsymbol{e}_{s}=0$ and $\boldsymbol{n} \cdot \boldsymbol{d}_{s}=0$, to neglect the field distorsion at a certain distance from $\Omega_{c, p}$.

Note that in regions where $\varepsilon_{p} \neq \varepsilon_{u}$, an additional source term given by the unperturbed solution $\left(\varepsilon_{p}-\varepsilon_{u}\right) \boldsymbol{e}_{u}$ is considered in (3 $\mathrm{k}$ ). Because the added region $\Omega_{c, p}$ is a perfect conductor, this source term is not directly involved. Actually, this region is extracted from $\Omega_{p}$ and treated via a $\mathrm{BC}$ of type 4 a) on its boundary $\Gamma_{c, p}$ (thus added to $\Gamma_{e, p}$ ), with

$$
\boldsymbol{n} \times\left.\boldsymbol{e}_{s}\right|_{\Gamma_{c, p}}=-\boldsymbol{n} \times\left.\boldsymbol{e}_{u}\right|_{\Gamma_{c, p}}
$$

or

$$
\left.v\right|_{\Gamma_{c, p}}=-v_{s} \text { with } v_{s}=\left.v_{u}\right|_{\Gamma_{c, p}} .
$$

In all the other regions, $\varepsilon_{p}$ and $\varepsilon_{u}$ are equal and denoted $\varepsilon$. 


\section{Weak Formulations}

\section{A. Unperturbed electric scalar potential formulation}

The unperturbed field distribution is first calculated in $\Omega$ as the solution of an electric scalar potential formulation, obtained from the weak form of $(3 \mathrm{~b})$, i.e., $\operatorname{div}\left(-\varepsilon \operatorname{grad} v_{u}\right)=0$, as

$$
\left(-\varepsilon \operatorname{grad} v_{u}, \operatorname{grad} v^{\prime}\right)_{\Omega}-\left\langle\boldsymbol{n} \cdot \boldsymbol{d}_{u}, v^{\prime}\right\rangle_{\Gamma_{d}}=0, \forall v^{\prime} \in F(\Omega)
$$

where $(\cdot, \cdot)_{\Omega}$ and $\langle\cdot, \cdot\rangle_{\Gamma}$ denote a volume integral in $\Omega$ and a surface integral on $\Gamma$ of the product of their arguments; $\mathrm{F}(\Omega)$ is the function space defined on $\Omega$ containing the basis functions for $v$ as well as for the test function $v^{\prime}[11]$. At the discrete level, $\mathrm{F}(\Omega)$ is approximated with nodal FEs. The surface integral term in (7) can be associated with a global quantity or used for fixing a natural BC (usually homogeneous for a tangent electric field constraint) on a portion $\Gamma_{d}$ of the boundary of $\Gamma$.

\section{B. Perturbation electric scalar potential formulation}

The source of the perturbation problem $v_{s}$ (6) is determined in the new added region $\Omega_{c, p}$ through a projection method [10]. Given the perfectly conductive nature of the perturbing region, the projection of $v_{u}$ from its original mesh to that of $\Omega_{c, p}$ can be limited to $\Gamma_{c, p}$. It reads

$$
\left\langle\operatorname{grad} v_{s}, \operatorname{grad} v^{\prime}\right\rangle_{\Gamma_{c, p}}-\left\langle\operatorname{grad} v_{u}, \operatorname{grad} v^{\prime}\right\rangle_{\Gamma_{c, p}}=0, \forall v^{\prime} \in F\left(\Gamma_{c, p}\right)
$$

where the function space $F\left(\Gamma_{c, p}\right)$ contains $v_{s}$ and its associated test function $v^{\prime}$. At the discrete level, $v_{s}$ is discretized with nodal FEs and is associated to a gauge condition fixing a nodal value in $\Gamma_{c, p}$.

Further, the projection is to be extended to the whole domain $\Omega_{c, p}$ in case of a dielectric perturbing region. We choose to directly project grad $v_{u}$ in order to assure a better numerical behaviour in the ensuing equations where the involved quantities are also gradients.

The perturbation electrostatic weak formulation in $\Omega_{p}$ is still of the form of 77 and reads

$$
\left(-\varepsilon \operatorname{grad} v, \operatorname{grad} v^{\prime}\right)_{\Omega_{p}}-\left\langle\boldsymbol{n} \cdot \boldsymbol{d}, v^{\prime}\right\rangle_{\Gamma_{d, p}}=0, \forall v^{\prime} \in F\left(\Omega_{p}\right)
$$

with non-homogeneous Dirichlet BC (6) and BCs (4a-b) in homogeneous forms.

When all components of the unperturbed electric field $\boldsymbol{e}_{u}=-\operatorname{grad} v_{u}$ are needed in the layer of FEs touching $\Gamma_{c, p}$ in $\Omega_{p} \backslash \Omega_{c, p}$ (to give access also to normal gradients), denoted $\Omega_{l, p}$, the projection (8) of $v_{u}$ has to be extended only to this transition layer. This way, the computational effort of the projection is also reduced. Having access to $\boldsymbol{e}_{u}$ and $\boldsymbol{e}$ in this layer allows to compute there the perturbed electric field $\boldsymbol{e}_{p}$. Both charges and electric forces can thus be calculated on $\Gamma_{c, p}$.

\section{Iterative sequence of perturbation problems}

For close relative positions where the coupling between the source and perturbing regions is significant, an accurate solution can be obtained via an iterative procedure that calculates successive perturbations not only from the initial source region to the added conductor but also from the latter to the former. Each region gives the suitable correction as a perturbation with an accuracy dependent of the fineness of its mesh.

For each iteration $i(i=0,1, \ldots)$, we determine the electric scalar potential $v_{2 i}$ in $\Omega$, starting with $v_{0}=v_{u}$. The projection of this solution from its original mesh to that of the added conductor $\Omega_{c, p}$ gives a source $v_{s, 2 i+1}$ for a 
perturbation problem. This way, we obtain a potential $v_{2 i+1}$ and an electric charge on $\Gamma_{c, p}$ that counterbalance the potential and the electric charge on $\Gamma_{c}$. For iteration $i+1$, a new source $v_{s, 2 i+2}$ for the initial configuration has to be then calculated. This is done by projecting $v_{2 i+1}$ from its support mesh to that of $\Omega$. This projection can be limited to the layer of FEs touching $\Gamma_{c}$ in $\Omega \backslash \Omega_{c}$, denoted $\Omega_{l}$, i.e.,

$$
\left(\operatorname{grad} v_{s, 2 i+2}, \operatorname{grad} v^{\prime}\right)_{\Omega_{l}}-\left(\operatorname{grad} v_{2 i+1}, \operatorname{grad} v^{\prime}\right)_{\Omega_{l}}=0, \forall v^{\prime} \in F\left(\Omega_{l}\right)
$$

A perturbation electric scalar potential problem is defined in $\Omega$ as

$$
\left(-\varepsilon \operatorname{grad} v_{2 i+2}, \operatorname{grad} v^{\prime}\right)_{\Omega}-\left\langle\boldsymbol{n} \cdot \boldsymbol{d}_{2 i+2}, v^{\prime}\right\rangle_{\Gamma_{d}}=0, \forall v^{\prime} \in F(\Omega),
$$

with non-homogeneous Dirichlet BC $v_{2 i+2}=-\left.v_{s, 2 i+2}\right|_{\Gamma_{c}}$ and Neumann BC $\left.\boldsymbol{n} \cdot \boldsymbol{d}_{2 i+2}\right|_{\Gamma_{d}}=-\left.\boldsymbol{n} \cdot \boldsymbol{d}_{2 i+1}\right|_{\Gamma_{d}}$. The latter is not known in a strong sense. The associated surface integral term in 110 can be evaluated rather via the weak formulation of problem $2 i+1$ now applied to $\Omega_{l}$, as

$$
\begin{aligned}
\left\langle\boldsymbol{n} \cdot \boldsymbol{d}_{2 i+2}, v^{\prime}\right\rangle_{\Gamma_{d}} & =-\left\langle\boldsymbol{n} \cdot \boldsymbol{d}_{2 i+1}, v^{\prime}\right\rangle_{\Gamma_{d}} \\
& =-\left(-\varepsilon \operatorname{grad} v_{s, 2 i+2}, \operatorname{grad} v^{\prime}\right)_{\Omega_{l}}, \forall v^{\prime} \in F\left(\Omega_{l}\right)
\end{aligned}
$$

benefiting from the projection $v_{s, 2 i+2}$ of $v_{2 i+1}$ already done by (10).

This iterative process is repeated until convergence for a given tolerance.

\section{Electrostatic charges}

A suitable treatment of the surface integral term in (9) consists in naturally defining a global electric charge in the weak sense [11]. A test function $v_{c, p}$ is chosen equal to one on $\Gamma_{c, p}$ and continuously varying towards 0 in the layer $\Omega_{l, p}$ of FEs touching $\Gamma_{c, p}$ in $\Omega_{p} \backslash \Omega_{c, p}$. The electric charge can be naturally calculated at the post-processing stage through the volume integral in (9) limited to $\Omega_{l, p}$, i.e.,

$$
Q_{p}=Q_{u}+Q
$$

with

and

$$
\begin{aligned}
Q_{u} & =-\left(-\varepsilon \operatorname{grad} v_{s}, \operatorname{grad} v_{c, p}\right)_{\Omega_{l, p}}, \\
Q & =-\left(-\varepsilon \operatorname{grad} v, \operatorname{grad} v_{c, p}\right)_{\Omega_{l, p}} .
\end{aligned}
$$

The calculation of $Q_{u}$ needs to use the unperturbed potential $v_{u}$ expressed in the mesh of $\Omega_{l, p}$, which is actually the projected potential $v_{s}$. This justifies the interest of projecting $v_{u}$ in $\Omega_{l, p}$.

In case of an iterative sequence of perturbation problems, the total charge on $\Gamma_{c, p}$ is given by the summation of correction charges obtained at each iteration $i$.

\section{E. Electrostatic forces}

As previously mentioned, the perturbed electric field $\boldsymbol{e}_{p}$ can be computed in the layer $\Omega_{l, p}$ of FEs touching $\Gamma_{c, p}$ in $\Omega_{p} \backslash \Omega_{c, p}$. The electric force distribution is calculated thus by locally applying the virtual work principle [13] in this transition layer. At the discrete level, the force at each node of $\Gamma_{c, p}$ is obtained by deriving the electric energy in the considered layer of FEs with respect to a virtual displacement. The contribution of a reference element $\Delta$ to the force in a given direction is

$$
F_{r}=\int_{\Delta} \frac{\varepsilon}{2}\left(-2 \boldsymbol{e}_{p} J^{-1} \frac{\partial J}{\partial u} \boldsymbol{e}_{p}+\boldsymbol{e}_{p} \boldsymbol{e}_{p} \frac{\partial|J|}{\partial u}\right) d \Delta
$$

for a virtual displacement $r$ in this direction. $J$ is the geometrical Jacobian matrix with determinant $|J|$. 
Given the non-linearity of the force, a direct summation of the forces at each iteration is not possible. The total electric field $\boldsymbol{e}_{p}$ has to be updated at each iteration before computing the total force by (14).

\section{Elastic FE Model}

A brief description of the elastic FE model of MEMS is given for a linear isotropic material. Under this hypothesis, the equation of static equilibrium of a body under volume forces $\mathbf{f}_{\mathbf{v}}$ reads [12]

$$
\nabla[\sigma]+\mathbf{f}_{\mathbf{v}}=0
$$

where $[\sigma]$ is the symmetric Cauchy stress tensor. Next, the stresses $\sigma_{i j}$ are related to strains $\epsilon_{i j}$ by the Hook law as following

$$
[\sigma]=[E][\epsilon]
$$

with $[E]$ the material stiffness tensor that depends on the Young modulus $E$ and the Poisson ratio $\nu$. The strain tensor $[\epsilon]$ is $\nabla \mathbf{u}$, where $\mathbf{u}=\left(u_{x}, u_{y}, u_{z}\right)^{T}$ are the mechanical displacements. From 15 , we can write the system characterizing the linear mechanical problem

$$
\nabla[E] \nabla \mathbf{u}+\mathbf{f}_{\mathbf{v}}=0
$$

An additional smoothening step is performed in order to distribute the mechanical displacement of the moving microstructure over the air domain $\Omega_{p}$. This smoothening can be seen as an additional mechanical equation of type (17) applied to the exterior region, with an arbitrary material stiffness $[E]$. The displacements of the deforming electrode are used as non-homogeneous Dirichlet boundary conditions and keep the rest of the domain $\Omega_{p}$ boundary fixed. After solving this equation, we update the nodal coordinates of the mesh of domain $\Omega_{p}$. Next, a new electrostatic problem by the PFEM is carried out. This sequential coupling is repeated until the relative change in nodal displacements of the moving microstructure is bounded by a given threshold $\alpha$

$$
\frac{\left\|\mathbf{u}_{\mathbf{i}+\mathbf{1}}-\mathbf{u}_{\mathbf{i}}\right\|_{2}}{\left\|\mathbf{u}_{\mathbf{i}+\mathbf{1}}\right\|_{2}} \leq \alpha
$$

with $\mathbf{u}_{\mathbf{i}+\mathbf{1}}$ is the displacement of the current iteration and $\|.\|_{2}$ is the Euclidean norm.

\section{Applications}

\section{A. Cantilever Microbeam}

The electromechanical analysis of a cantilever microbeam (Fig. 3) is first carried out in order to illustrate and validate the PFEM. The perturbing conductive part $\Omega_{c, p}$ is a flexible microbeam $\left(L_{1}=175 \mu \mathrm{m}, t=1.85 \mu \mathrm{m}\right)$ suspended over a stationary electrode $\Omega_{c}\left(L_{2}=168 \mu \mathrm{m}\right)$. The air gap $g$ (i.e., distance between both conductors) is $2 \mu \mathrm{m}$. The Young modulus $E$ of the polysilicon cantilever microbeam is $135 \mathrm{GPa}$ and the Poisson ratio $\nu$ is 0.22 .

The domain $\Omega$ surrounding the fixed electrode $\Omega_{c}$ is coarsely meshed (Fig. $4(a)$ ). The domain $\Omega_{p}$ containing the deforming microstructure $\Omega_{c, p}$ (i.e., the structural domain $\Omega_{M}$ ) has an adapted mesh especially fine in the vicinity of the corners (Fig. $4(b)(c)$ ). The fringing field effects are then taken into account and the electric forces are accurately calculated. Further, the two meshes are independent what allows for any intersection of perturbation problem boundaries with the unperturbed problem material regions. Degenerated elements of the electric domain are then avoided in case of critical deformation of the microbeam subjected to a voltage near to the pull-in value. 
The maximum vertical microbeam deflection (in micrometers) versus potential difference between the deforming plate and the ground is depicted in Fig. 5] The results obtained with the PFEM are compared with measurements [15] and the classical FEM. On the one hand, one can see that there is a good agreement between PFEM results and experimental ones. On the other hand, the curves obtained with the PFEM and classical FEM agree well for low applied voltage, when the deformation is small. When the voltage increases, the displacement is critical and the PFEM curve deviates from the FEM one but agrees well with the experimental result. That is because the mesh of the classical FE model around the corners is not as fine as the one of the perturbation model. In fact, it is not fine enough. Indeed, a finer mesh of the FEM model would lead to degenerated elements for large displacements of the deflecting microbeam. The experimental pull-in voltage is $14.5 \mathrm{~V}$ whereas the ones predicted by the PFEM and the conventional FEM are $14.35 \mathrm{~V}$ and $15.35 \mathrm{~V}$, respectively ( $1 \%$ and $6 \%$ as relative error with respect to measurement, respectively).

\section{B. Combdrive}

In order to illustrate the PFEM when applied to the electrostatic analysis of MEMS with a rigid movement, a combdrive is considered. Its geometry is shown in Fig. 6 ( $L=10 \mu \mathrm{m}, b=2 \mu \mathrm{m}$ and $g=2 \mu \mathrm{m})$. The movement of the finger is simulated by varying its engagement $x$.

The domain $\Omega$ surrounding the fixed comb is coarsely meshed (Fig.7 (right)). The domain $\Omega_{p}$ containing the moving finger (i.e., $\Omega_{c, p}$ ) has an adapted mesh especially fine in the vicinity of the corners (Fig. 7 (left)). The two meshes are independent.

At odd iterations, the electric charges appearing on the surface of the moving finger for each of its positions are calculated. Fig. 8 shows the convergence of the charges as a function of iteration $i$. As expected, more iterations are required when the moving finger is close to the stationary electrode (21 iterations at $x=3 \mu \mathrm{m}, 11$ iterations at $x=9 \mu \mathrm{m}$ ). Nevertheless, using the Aitken acceleration [16] allows the iteration process to be more efficient. In this case, only 5 iterations are needed for obtaining the charges in each position of the moving finger.

Further, the numerical results of the electric charges and forces ( $x$-component) obtained by the PFEM with Aitken acceleration and the conventional FEM are compared in Fig. 9 . These quantities are determined with only 5 iterations by the PFEM. A good agreement is observed (the relative error with respect to the FEM results reaches $5 \%$ ). In addition, it has been verified that the PFEM solves this 2-D problem with a speed-up factor between 2 and 3 in comparison to the classical FEM.

\section{Conclusion}

The analysis of the coupled electromechanical problem of electrostatic MEMS has been investigated by an iterative perturbation finite element technique. It has been shown to be of interest for overcoming degenerated elements when some moving regions undergo critical deformations. The analysis of a cantilever microbeam has been carried out. The accuracy of the proposed method has been demonstrated by comparing the results with both measurements and the classical FEM calculations. The electrostatic analysis of a combdrive involving rigid movement has also been performed. The Aiken acceleration has been proven to be very efficient. A significant speed-up in comparison to the conventional FEM is achieved.

\section{Acknowledgments}

This work was supported by the Belgian French Community (ARC 03/08-298) and the Belgian Science Policy (IAP P6/21). 


\section{References}

1. Gilbert, J. R., Ananthasuresh, G. K., Senturia, S. D. “3D modeling of contact and hysteresis in coupled electro-mechanics," MEMS'96, 1996, pp. $127-132$.

2. Gyimesi, M., Avdeev, I., Ostergaard, D., "Finite-Element Simulation of Micro-Electromechanical Systems (MEMS) by Strongly Coupled Electromechanical Transducers," IEEE Trans. Magn., Vol. 40, No. 2 (2004), pp. 557-560.

3. Zhulin, V.I., Owen, S.J., Ostergaard, D.F., "Finite Element Based Electrostatic-Structural Coupled Analysis with Automated Mesh Morphing," MSM 2000, the International Conference on Modeling and Simulation and Simulation of Microsystems, San Diego, March 2000 , pp. 501-504.

4. Perrin-Bit, R. and Coulomb, J.L., "Three Dimensional Finite Element Mesh Connection for Problems Involving Movement," IEEE Trans. Magn., Vol. 31, No. 3 (1997), pp. 1920-1923.

5. Aluru, N.R. and White, J., "Direct Newton Finite-Element/Boundary-Element Technique For Micr-Electro-Mechanical-Analysis," Solid-State Sensor and Actuator Workshop, Hilton Head Island, June 1996, pp. 54-57.

6. Farina, M. and Rozzi, T., “A Galerkin Projection Method for Mixed Finite Elements," IEEE Trans. on Microwave Theory and Techniques, Vol. 49, No.12 (2001), pp.2235-2240.

7. Badics, Z. et al., "An Effective 3-D Finite Element Scheme for Computing Electromagnetic Field Distortions due to Defects in Eddy-Current Nondestructive Evaluation," IEEE Trans. Magn., Vol. 33, No. 2 (1997), pp. 1012-1020.

8. Dular, P. and Sabariego, R.V., "A Perturbation Finite Element Method for Modeling Moving Conductive and Magnetic Regions without Remeshing," COMPEL, The international journal for computation and mathematics in electrical and electric engineering, Vol. 26, No. 3 (2007), pp. 700-711.

9. Boutaayamou, M., Sabariego, R.V., Dular, P., “An Iterative Finite Element Perturbation Method for Computing Electrostatic Field Distortions," IEEE Trans. Magn., 2008, in press.

10. Geuzaine, G., Meys, B., Henrotte, F., Dular, P., Legros, W., “A Galerkin Projection Method for Mixed Finite Elements," IEEE Trans. Magn., Vol. 35, No. 3 (1991), pp. 1438-1441.

11. P. Dular, W. Legros, A. Nicolet, "Coupling of local and global quantities in various finite element formulations and its application to electrostatics, magnetostatics and magnetodynamics," IEEE Trans. Magn., vol. 34, no. 5 (1998), pp. 3078-3081.

12. Pelesko, J. A. and Bernstein, D. H., "Modeling MEMS and NEMS," Chapman and Hall (London, 2002).

13. Coulomb, J. L. and Meunier, G., "Finite Element Implementation of Virtual Work Principle for Magnetic or Electric Force and Torque Computation," IEEE Trans. Magn., Vol. 20, No. 5 (1984), pp. 1894-1896.

14. Imhoff, J. F., Meunier, G., Brunotte, X., Sabonnadière, J. C., "An original solution for unbounded electromagnetic 2D and 3D problems throughout the finite element method," IEEE Trans. Magn., Vol. 26, No. 5 (1990), pp. 1629-131.

15. Rochus, V., "Finite element modelling of strong electro-mechanical coupling in MEMS," Ph.D. dissertation, University of Liège (Belgium, 2006).

16. Weniger, E. J., "Prediction properties of Aitken's iterated $\delta^{2}$ process, of Wynn's epsilon algorithm and of Brezinski's iterated theta algorithm," J. Comput. Appl. Math., Vol. 122, No. 1-2 (2000), pp. 329-356. 


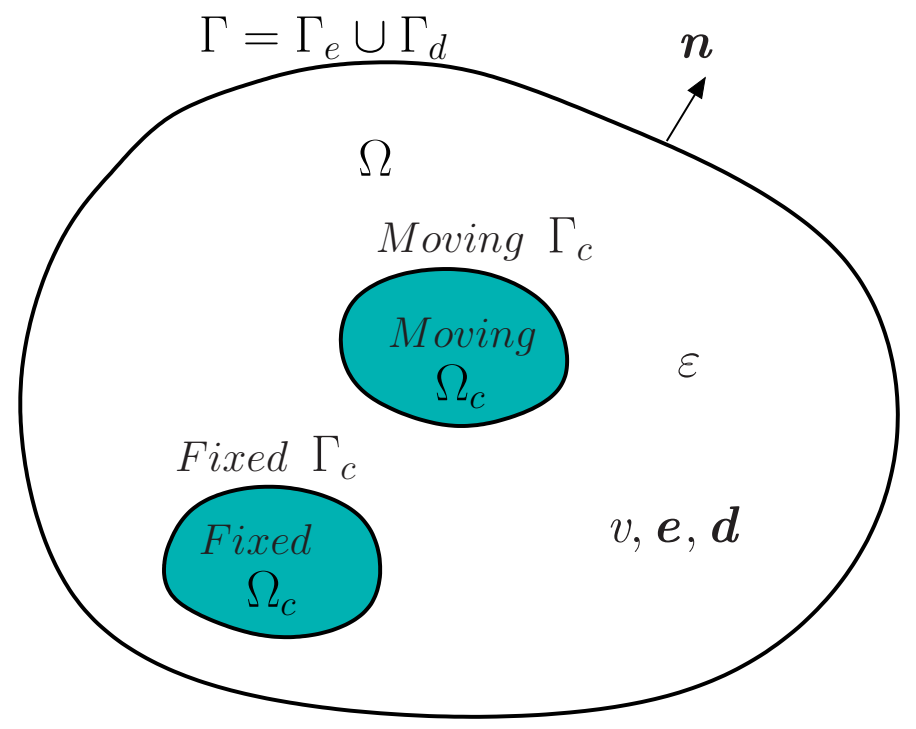

Fig. 1

Bounded Domain $\Omega$ OF THE 2-D OR 3-D EUCLIDEAN SPACE With BOUNDARY $\Gamma=\Gamma_{e} \cup \Gamma_{d}$.

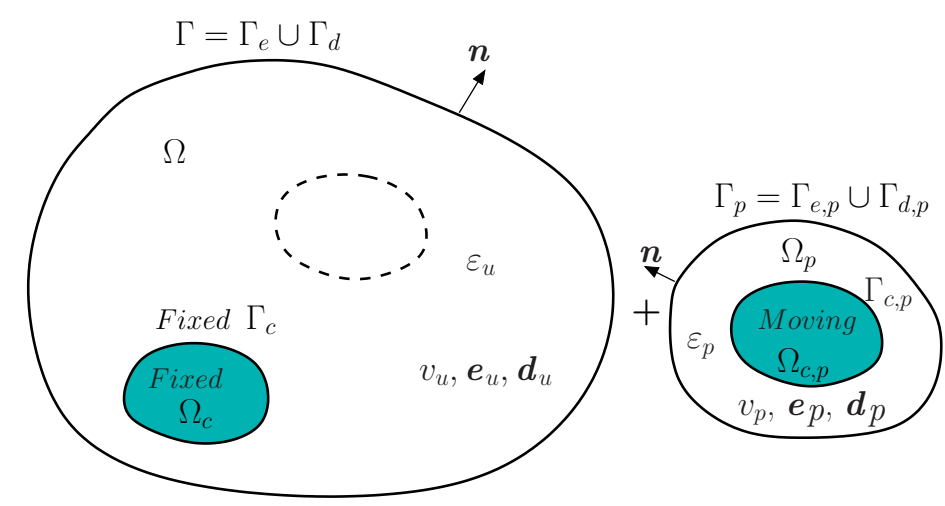

Fig. 2

DeComposition of the Classical electric PRoblem deFined in Domain $\Omega$ (Fig. 1 into unPerturbed (left) AND PERTURbation (right) PROBLEMS. 


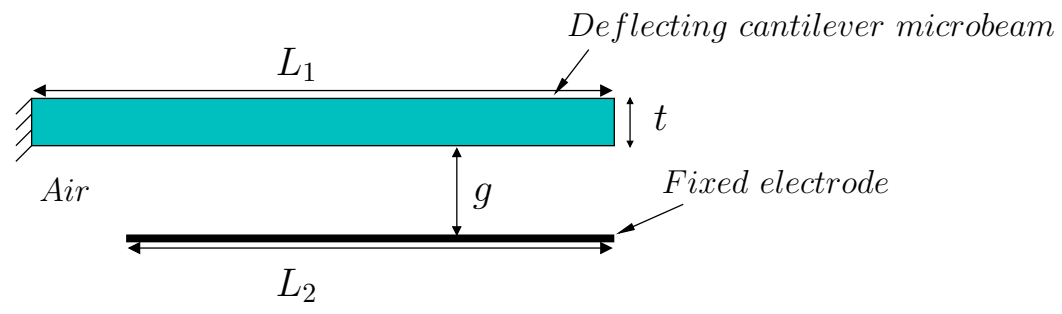

Fig. 3

GEOMETRY OF THE CANTILEVER MICROBEAM.

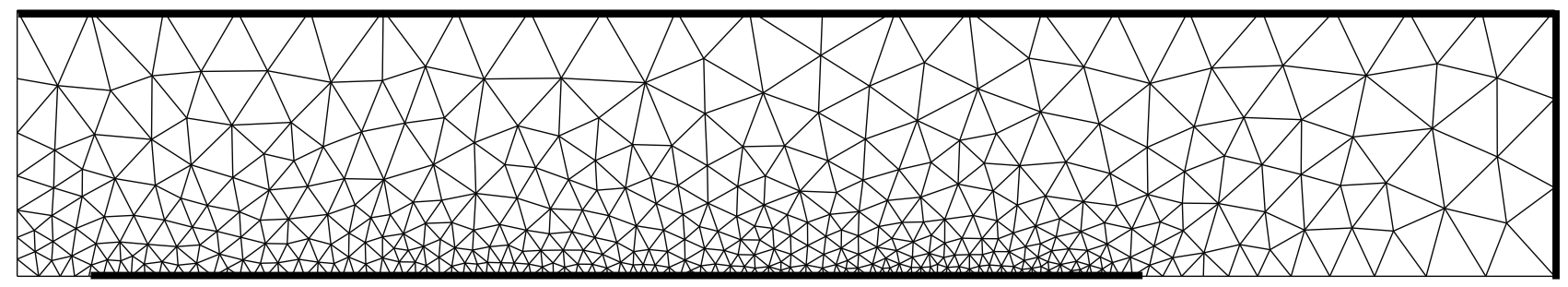

(a)

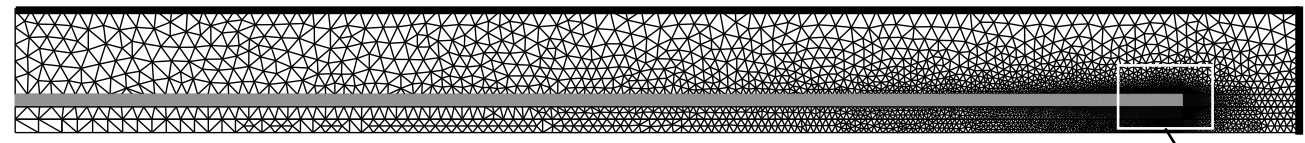

(b)

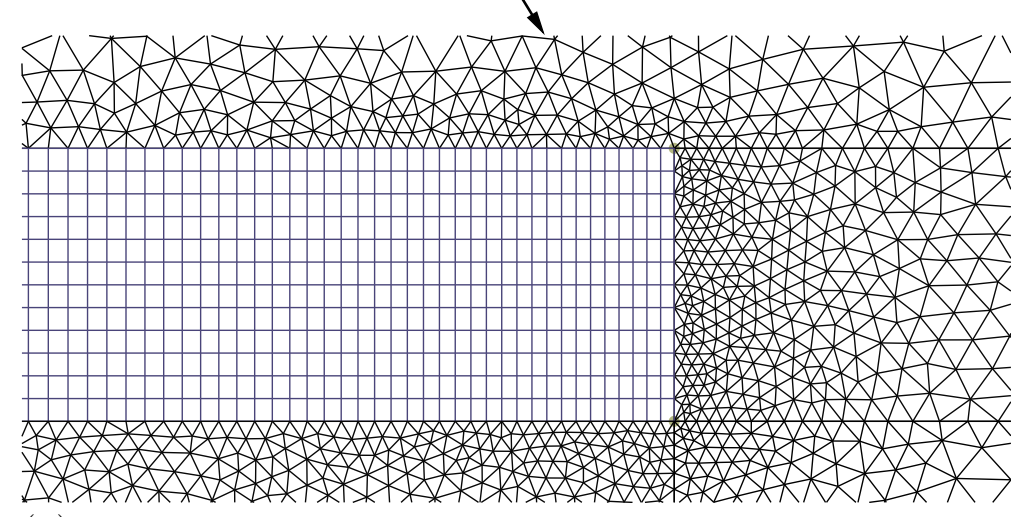

$(c)$

Fig. 4

Mesh of the UnPerturbed $(a)$ AND Perturbed $(b)$ Domains With infinite boundaries [14] AND A Very Fine Mesh $(c)$ IN THE VICINITY OF THE CORNERS OF THE PERTURBING CANTILEVER MICROBEAM TO ACCOUNT FOR THE FRINGING FIELD EFFECTS. 


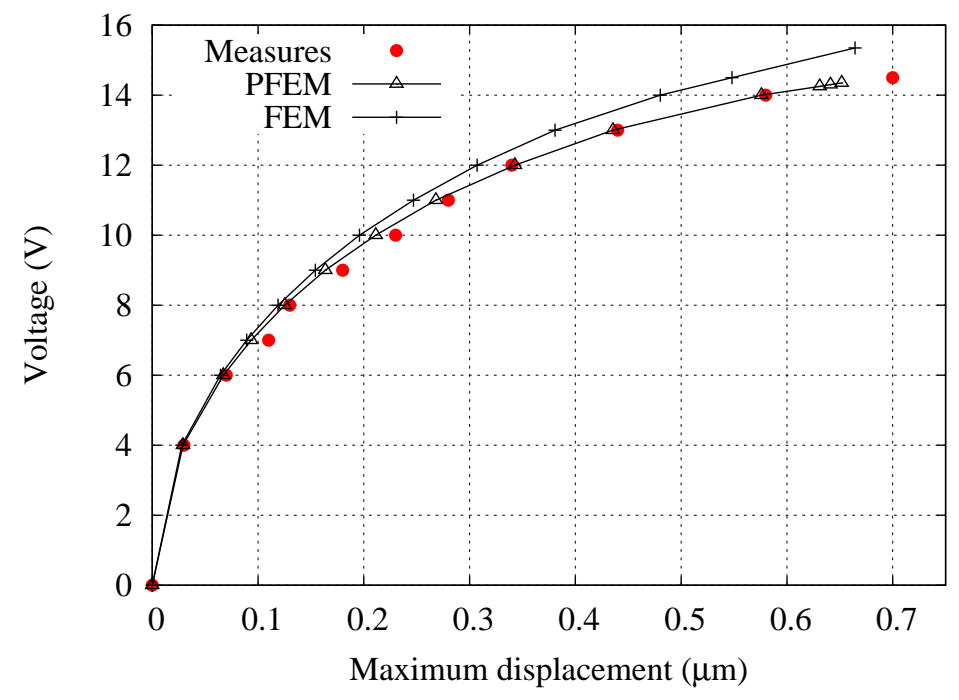

Fig. 5

COMPARISON BETWEEN EXPERIMENTAL MAXIMUM TIP DISPLACEMENT OF A CANTILEVER BEAM SUBJECTED TO AN ELECTRIC VOLTAGE WITH THE FEM AND PFEM NUMERICAL RESULTS.

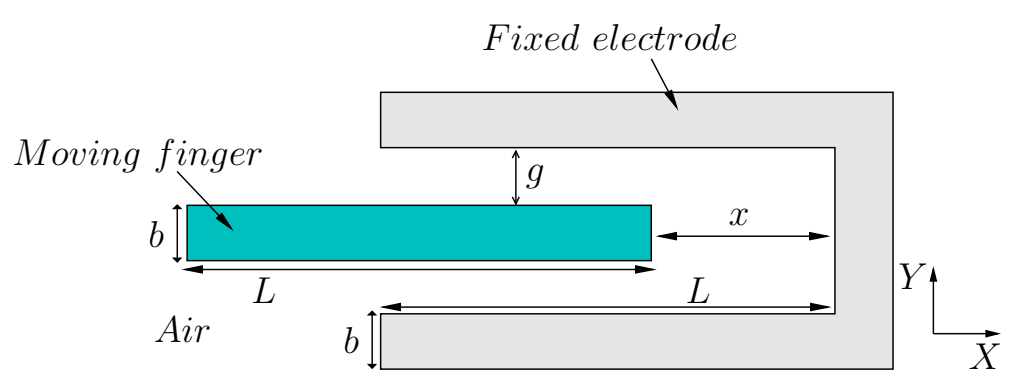

Fig. 6

GEOMETRY OF THE COMBDRIVE.
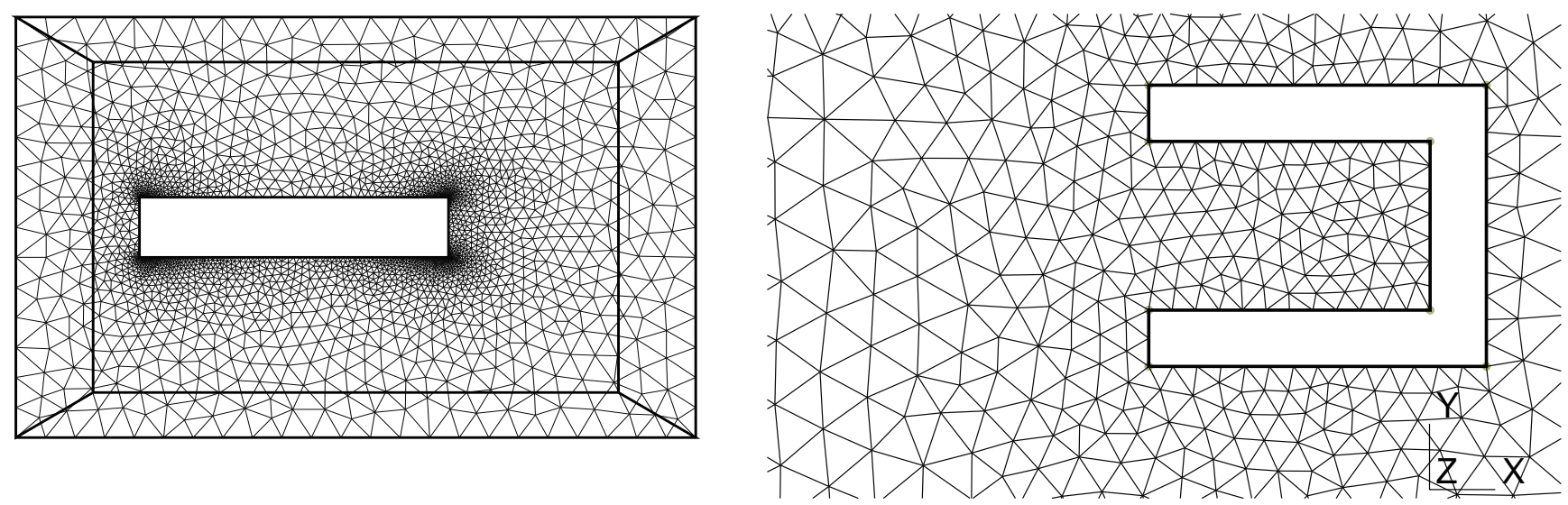

Fig. 7

MESH OF $\Omega(r i g h t)$ AND ADAPTED MESH OF $\Omega_{p}$ WITH INFINITE BOUNDARIES AROUND THE MOVING FINGER (left). 


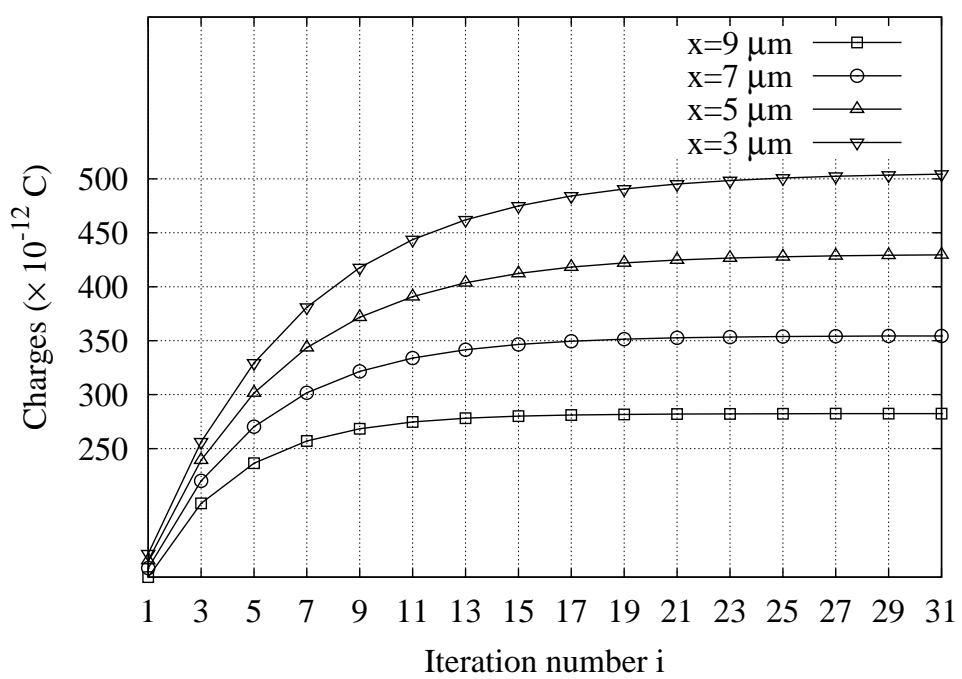

Fig. 8

ELECTRIC CHARGES CALCULATED BY THE PFEM ON THE MOVING FINGER FOR EACH POSITION $x$ AS A FUNCTION OF ITERATION NUMBER $i$ (Without AitKen ACCELERATion).
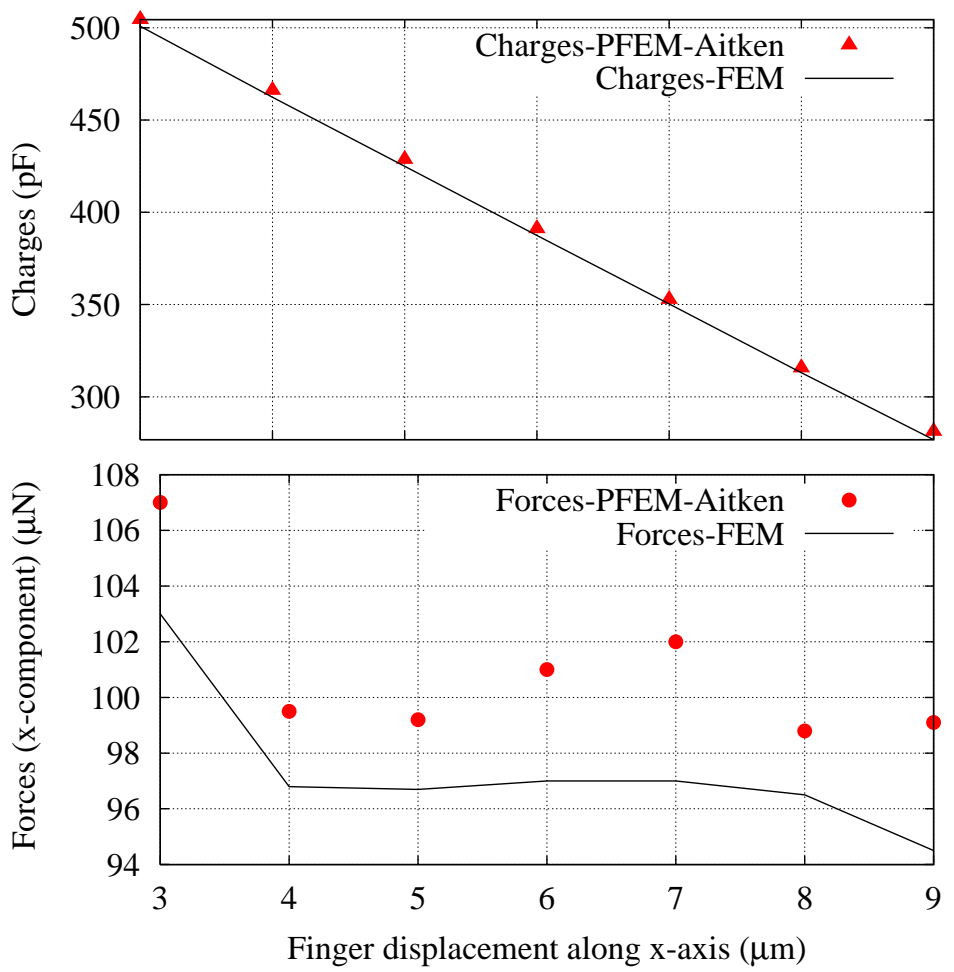

Fig. 9

ELECTRIC CHARGES (up) AND FORCES (down) VERSUS FINGER DISPLACEMENT. 